It is also important to note another aspect of this model. At the very high temperatures, $T \geq 10^{18}{ }^{\circ} \mathrm{K}$, at which these reactions occur, pair production of baryons may increase the density substantially. Under these conditions the Fermi temperature $T_{f} \approx 10^{-13} T^{2}\left(M_{n} / M_{b}\right)$, where $M_{n}$ is the neutron and $M_{b}$ the baryon mass. Thus neutrons will not become degenerate until $T \approx 10^{13}{ }^{\circ} \mathrm{K}$. If quarks are to combine at this or higher temperatures, the quark-baryon mass defect would have to be sufficiently large to release a Fermi energy of about $10^{-26} T^{2}\left(M_{n} / M_{b}\right)$ $\mathrm{GeV}$. Another effect which may become important at high densities is induced if the effective quark radius is much smaller than that of the baryon. In order to create a space $\Delta v$ for the newly formed baryon, an amount of energy approximately equal to the work $p \Delta v$ must be liberated, where $p$ is the local pressure. Were the mass defect unable to supply the required energy, quark combination would have to be balanced by processes which reduced the local pressure. For quark masses of the order of a few $\mathrm{GeV}$, this also becomes important at temperatures of about $10^{13}{ }^{\circ} \mathrm{K}$ and such quarks would not be expected to combine at earlier phases of the expansion, except possibly to form a statistical equilibrium with baryons. At these high temperatures there may also be pair production and annihilation of quarks. Such considerations provide relations between quark mass and temperature, and the resultant time-scales for evolution will also be affected by anisotropy and inhomogeneities.

If quark combination from an initially cold universe is responsible for the radiation field, the effects of degeneracy and pair production are less important. In an oscillating universe, the role of quarks may be to exchange energy between the matter and the radiation field during dense phases. Such an exchange, if irreversible, could lead to a damping of the oscillations. The discovery of quarks and their properties may well provide further constraints on theories of the early stages of an expanding universe.

It is a pleasure to thank Dr. R. J. Tayler, Dr. D. W. Sciama and Dr. S. W. Hawking for helpful advice. This work was done during the tenure of a Fulbright fellowship which I gratefully acknowledge.

WIILIAM C. SAStaw

Department of Applied Mathematies and

Theoretical Physics,

University of Cambridge.

'Zeldovic, Ya. B., et al., Phys. Lett., 17, 164 (1965).

'Bowen, T., et al., Phys. Rev. Lett., 18, 728 (1964).

${ }^{3}$ Sunyar, A. W., et al., Phys. Rev., 136, B1157 (1964).

- Penzias, A. E, and Wilson, R. W., Astrophys. J., 142, 419 (1965).

'Roll, P. G., and Wilkinson, D. T., Phys. Rev. Lett., 16, 405 (1965).

- Field, G. B., and Hitchcock, J., Phys. Rev. Lett, 16, 817 (1966).

- Thaddeus, P., and Clauser, J. F., Phys. Rev. Lett., 16, 819 (1966).

- Howell, T. F., and Shakeshaft, J. R., Nature, 210, 1318 (1966). - Gamow, G., Vistas in Astronomy, edit. by Beer, A., 2, 1726 (Pergamon
Press, London and New York, 1956).

'* Dicke, R. H., et al., Astrophys. J., 142, 414 (1965).

11 Peebles, P. J. E., Phys. Rev. Lett., 16, 410 (1966).

${ }^{12}$ Oort, J. H., Onzieme Conseil de I'Institut International de Phisique Solvay, La Structure et L'Evolution de L'Univers, 163 (Stoops, Brussels, 1958).

1* Hawking, S. W., and Tayler, R. J., Nature, 209, 1278 (1968).

14 Domokos, G., and Fulton, T., Phys. Lett., 20, 546 (1966).

${ }^{26}$ Woolf, N. J., Conf. on Observational Aspects of Cosmology, Miami (1965).

\section{THE SOLID STATE}

\section{Simultaneous Magneto-optic Recording of Hysteresis Loops from Both Surfaces of a Magnetic Film}

Magnetization reversal processes in thin magnetic films have been extensively investigated in recent years. The usual techniques adopted for the display of hysteresis loops have been based on the use of a pick-up coil to detect $\mathrm{d} M / \mathrm{d} t$, as described in detail by Crittenden et al..$^{1,2}$.

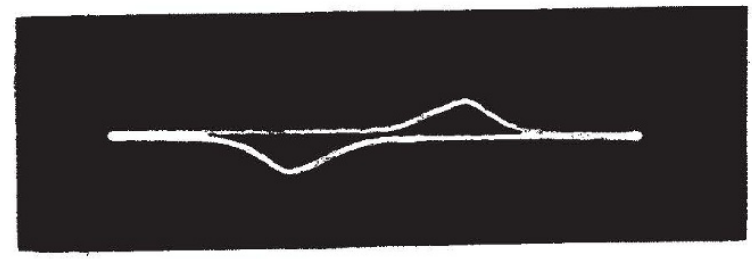

Fig. 1

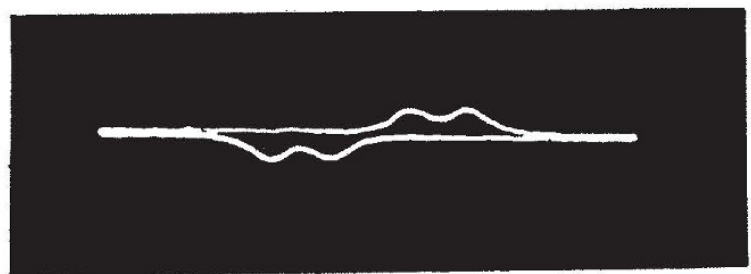

Fig. 2

More recently, use has been made of the magneto-optic effects and, in particular, Green and Thomas ${ }^{3}$ have developed a simple technique in which hysteresis loops are obtained using the transverse Kerr effect in the infra-red. With a $50 \mathrm{c} / \mathrm{s}$ drive field reversing the magnetization, the intensity changes in the reflected beam are detected by a phototransistor and then amplified.

This technique is being used in investigations of cobalt films in the thickness range $500-4000 \AA$ produced by evaporation on to glass substrates $(10-20 \AA / \mathrm{sec})$ and some of the results have already been reported elsewhere ${ }^{4}$. The simplicity of the method has now permitted simultaneous investigations of the magnetization changes occurring at both surfaces of a single film.

In the case of the thinner films $(<2000 \AA)$ the signals detected from each side appear identical. For the thicker films, however, differences are apparent in the signals obtained from each side. This effect first becomes noticeable at a thickness of about $2000 \AA$ and is very pronounced at about $4000 \AA$. Figs. 1 and 2 show the signals of $\mathrm{d} M / \mathrm{d} t$ versus drive field taken from the free surface and the substrate surface respectively of a $4000 \AA$ film. It is clear that the reversal process is different at each surface and, furthermore, preliminary results indicate that the effect is dependent on the orientation of the film with respect to the drive field.

Simultaneous recording in this fashion should find useful application in investigations of multiple films.

One of us (B. W. J. T.) is indebted to the Directors of International Computers and Tabulators (Engineering), Ltd., for their continued support.

R. CAREY

E. D. ISAAC

B. W. J. Thomas

Department of Applied Physics,

Lanchester College of Technology,

Coventry.

'Crittenden, Jun., E. C., Smith, Jun., C. S., and Olsen, L. O., Rev. Sei. Instrum., 17, 372 (1946).

- Crittenden, jun., E. C., Hudimac, A. A., and Strough, R. I., Rev. Sei. Instrum., 22, 873 (1951).

Green, A., and Thomas, B. W. J., J. Sci. Instrum., 48, 399 (1966). - Carey, R., Isaac, E. D., and Thomas, B. W. J., Brit. J. App. Phys., 17,
635 (1966).

\section{Synthetic Single Crystals of Graphite}

THE formation of Kish graphite in iron/carbon is well known ${ }^{1}$, and reports have appeared of the growth of usable single crystals of graphite at $3,200^{\circ} \mathrm{C}$ in these systems ${ }^{2}$. Such crystals have not had the benefit of formation at a high temperature, so that in order to produce 